\title{
Uso do óleo de pequi (Caryocar brasiliense) em emulsões cosméticas: desenvolvimento e avaliação da estabilidade física
}

\author{
Aline Rocha Pianovski, Adrea Fernanda Gonçalves Vilela, Alex Antonio Serafim da Silva, \\ Cleide Garbelini Lima, Ketlyn Konageski da Silva, Vanessa Franco Marcelo Carvalho, \\ Carlo Ralph De Musis, Silvia Regina Pengo Machado, Márcio Ferrari*
}

Faculdade de Farmácia, Universidade de Cuiabá

*Correspondência:

M. Ferrari

Laboratório de Pesquisa e

Desenvolvimento de Produtos

Cosméticos

Universidade de Cuiabá - UNIC

Av. Beira Rio, 3100. Jardim Europa 78015-480 - Cuiabá - MT, Brasil

E-mail: ferrarimarcio@uol.br
Os objetivos deste trabalho foram desenvolver e avaliar a estabilidade física de emulsões $O / A$ contendo óleo de pequi (Caryocar brasiliense). Emulsões O/A contendo 10,0\% (p/p) de óleo de pequi foram preparadas e, para promover a estabilidade, a adição de carbomer, magnesium sulfate, sodium chloride e sorbitan oleate, foram estudadas. O tipo de emulsão foi verificado pelo método de diluição e o aspecto, homogeneidade e características organolépticas avaliadas através de análises macroscópicas. Como testes preliminares foram utilizados a centrifugação, ciclo gela-degela e o estresse térmico. Para avaliar a estabilidade acelerada as amostras foram submetidas em diferentes condições de estresse e analisadas a partir do valor de $p H$, análises macroscópicas e comportamento reológico. As emulsões preparadas com óleo de pequi, 0,3\% (p/p) de Acrylates/ C10-30 Alkyl Acrilate Crosspolymer e 0,2\% (p/p) de carbomer apresentaram-se estáveis com propriedades pseudoplásticas e tixotrópicas. As características macroscópicas e valores obtidos de $\mathrm{pH}$, viscosidade aparente, indices de fluxo e de consistência da área de histerese durante a estocagem indicaram estabilidade da formulação.
Unitermos

- Caryocar brasiliense

- Emulsão O/A

- Óleo de pequi

- Reologia

- Teste de estabilidade

\section{INTRODUÇÃO}

Emulsão é um sistema termodinamicamente instável resultante da mistura de dois líquidos imiscíveis entre si e uma terceira fase contendo agente emulsificante (Breuer, 1985).

De acordo com a natureza da fase externa, contínua ou ainda conhecida como dispersante podem ser classificadas em: emulsão água em óleo $(\mathrm{A} / \mathrm{O})$ a que contém água como fase dispersa sob a forma de pequenas partículas (maior que $0,1 \mathrm{~mm}$ ) na fase oleosa e óleo em água $(\mathrm{O} / \mathrm{A})$ a emulsão composta pela dispersão de material oleoso/graxo na fase aquosa (Sharma, Shah, 1985).

São muito utilizadas em cosméticos, para aplicação tópica, assim como em preparações farmacêuticas administradas por diferentes vias de administração (Pinho, Storpirtis, 1998), podendo ser incorporados em suas fases 
ativos hidrossolúveis e/ou lipossolúveis dependendo de suas características e dos efeitos desejados (Allen Junior, 2004).

Segundo a Farmacopéia Americana (USP, 1990) estabilidade é definida como a amplitude na qual um produto mantém dentro de limites especificados, as mesmas propriedades e características que possuía quando de sua fabricação durante o seu período de armazenamento e uso.

A instabilidade de uma emulsão pode se manifestar das seguintes formas (Silva, Soares, 1996): cremosidade, onde as partículas menos densas sobem para o topo da formulação; floculação: a força de repulsão entre as moléculas é diminuída e elas se associam de maneira fraca e reversível por agitação; coalescência: as gotículas da fase interna se unem e formam uma outra gotícula sendo este processo irreversível; e a inversão quando ocorre a inversão de fase, a externa torna-se interna e vice-versa.

À medida que as emulsões se tornam instáveis, suas características físico-químicas variam. Para verificar tais variações pode-se determinar o valor do $\mathrm{pH}$, viscosidade, densidade, condutividade elétrica, umidade, tamanho da partícula, entre outros (ANVISA, 2004).

As emulsões cosméticas e farmacêuticas são sistemas complexos, polidispersos contendo diferentes tensoativos de natureza química diversa e outros aditivos. A presença de filmes interfaciais estáveis já não pode ser considerada como o papel mais importante na estabilidade, mas outros mecanismos, entre os quais se destaca a formação de uma estrutura da fase contínua, que forma uma barreira reológica, evitando o movimento das gotículas (Ribeiro, 2006). De acordo com o mesmo autor, os tensoativos podem agir das seguintes formas para estabilizar as emulsões: estabilização eletrostática, estérica, por partículas sólidas e por misturas de emulsionantes.

Qualquer componente presente na formulação pode alterar sua estabilidade, sendo estes considerados fatores intrínsecos. As incompatibilidades físicas e químicas, incluindo nesta última, $\mathrm{pH}$, reações de oxi-redução e hidrólise, interação entre os componentes da formulação e com o material de embalagem, são consideradas fatores intrínsecos. Fatores extrínsecos relacionados aos materiais de embalagem, processo de fabricação, condições ambientais e de transporte também poderão influenciar na estabilidade (ANVISA, 2004).

$\mathrm{Na}$ área cosmética não existe nenhum protocolo oficial padronizando os testes de estabilidade, pois estes devem ser adequados aos objetivos do formulador, da forma cosmética e dos constituintes da formulação. No entanto, com o intuito de direcionar as indústrias cosméticas e/ou os formuladores, a Agência Nacional de Vigilância Sanitária (ANVISA), publicou um Guia de estabilidade sugerindo parâmetros de avaliação e os testes de estabilidade (ANVISA, 2004).

Segundo este Guia, os testes podem ser classificados de acordo com as seguintes etapas: centrifugação, se aprovado segue para os testes seguintes denominados preliminares, de triagem, ou ainda de curto prazo, tendo duração de aproximadamente 15 dias. Os testes de estabilidade normal ou exploratório e também chamado de estabilidade acelerada têm duração aproximada de 90 dias. Além destes, recomenda-se realizar o teste de prateleira, também denominado de longa duração ou shelf life que acompanha todo o tempo de validade do produto.

A Caryocar brasiliense (pequi) é uma árvore originária do Brasil (Collevati et al., 2003; Franquilino, 2006) de onde é extraído o óleo de interesse cosmético. Na composição do óleo de pequi verifica-se a presença da vitamina A e de diversos ácidos graxos como o palmítico, oléico, mirístico, palmitoléico, esteárico, linoléico e linolênico (Croda do Brasil, 2002; Facioli, Gonçalves, 1998). A presença destes ácidos graxos na pele é fundamental para manutenção da hidratação cutânea, da barreira cutânea e do manto hidrolipídico (Rieger, 1987).

Azevedo-Meleiro e Rodriguez-Amaya (2004) identificaram carotenóides no pequi Caryocar brasiliense. Estes metabólitos conferem proteção à pele impedindo a lipoperoxidação, evitando desta maneira a formação de radicais livres e conseqüentemente retardando envelhecimento cutâneo. Também foram demonstradas as atividades leishmanicida, antimicrobiana do extrato das folhas de pequi (Paula-Junior et al., 2006).

Sendo assim, é de grande importância o estudo da utilização do óleo de pequi, vislumbrando a aplicabilidade na área cosmética, sinalizando o aproveitamento de recursos naturais com desenvolvimento sustentável e consequentemente desenvolvimento regional e contribuição social.

Os objetivos deste trabalho foram desenvolver e avaliar a estabilidade física de emulsões cosméticas $\mathrm{O} / \mathrm{A}$ contendo óleo vegetal do cerrado brasileiro contemplado na flora mato-grossense: óleo de pequi.

\section{MATERIAL E MÉTODOS}

As emulsões foram formuladas com as seguintes matérias-primas denominadas pela International Nomenclature Cosmetics Ingredients (INCI) (ICID, 2000): Acrylates/C10-30 Alkyl Acrilate Crosspolymer e Carbomer (BFGoodrich, Dinaco SA), Caryocar brasiliense fruit oil, Caprylic/ Capric Triglyceride, Oleyl Alcohol (Croda do Brasil Ltda), BHT, Dissodium EDTA, Magnesium Sulfate, Sodium Chloride $(\mathrm{NaCl})$, Triethanolamine (Henrifarma Ltda), Methyldibromo 
Glutaronitrile (and) phenoxyethanol (Galena Química e Farmacêutica Ltda), Sorbitan Oleate (Oxiteno), Parfum e Aqua.

\section{Preparo da emulsão}

Foram preparadas pelo método de inversão de fases. As fases aquosa e oleosa foram aquecidas a $75,0^{\circ} \mathrm{C}$. Sob agitação constante adicionou-se o Acrylates/C10-30 Alkyl Acrilate Crosspolymer na fase oleosa permanecendo por 1 hora até total dispersão, vertendo posteriormente a fase aquosa. Foi mantida a agitação a $1200 \mathrm{rpm}$ durante 25 minutos. Após este tempo, foi adicionado o Methyldibromo Glutaronitrile (and) phenoxyethanol (Ferrari, 2002; Pemulen, 1999). Todas as formulações foram preparadas e avaliadas em 3 lotes diferentes. Emulsões aditivadas com Carbomer, Magnesium Sulfate, Sodium Chloride e Sorbitan oleate, associados ou não, também foram avaliadas (Tabela I).

\section{Análise macroscópica das formulações}

Realizada após $24 \mathrm{~h}$ do preparo das amostras e durante todas as avaliações, observou-se as características organolépticas e a homogeneidade das formulações (Ferrari, 1998).

\section{Determinação do tipo de emulsão}

O teste de diluição foi realizado com o objetivo de identificar o tipo de emulsão (A/O ou O/A) (Davis, 1977).

\section{Testes preliminares de estabilidade}

Teste de centrifugação

Em tubo de ensaio cônico graduado para centrífuga (Fanem Ltda-Mod. 206 R, Excelsa BABY II-440 watts) foram adicionados $10,0 \mathrm{~g}$ de cada amostra, pesados em balança semi-analítica (Gehaka, Mod. BG 2000) e submetidas aos ciclos de 1000, 2500 e $3500 \mathrm{rpm}$ (70, 440 e 863 $\mathrm{g}$, respectivamente) durante quinze minutos em cada rotação à temperatura ambiente (Ferrari, 1998; Idson, 1988; Idson, 1993a, 1993b; Rieger, 1996).

\section{Estresse Térmico}

As emulsões testes, foram submetidas ao aquecimento em banho termostatizado (Nova Ética Ltda-Mod. 500/

TABELA I - Composição das emulsões O/A com óleo de pequi.

\begin{tabular}{|c|c|c|c|c|c|c|c|c|c|c|}
\hline$\overline{\text { COMPOSIÇÃO }}$ & $\begin{array}{l}\mathrm{P} 1 \% \\
(\mathrm{p} / \mathrm{p}) \\
\end{array}$ & $\begin{array}{l}\mathrm{P} 2 \% \\
(\mathrm{p} / \mathrm{p}) \\
\end{array}$ & $\begin{array}{c}\text { P3, P4, } \\
\text { P5\% } \\
\text { (p/p) }\end{array}$ & $\begin{array}{c}\text { P6, P7, } \\
\text { P8\% } \\
(\mathrm{p} / \mathrm{p}) \\
\end{array}$ & $\begin{array}{c}\mathrm{P} 9- \\
\mathrm{P} 10 \% \\
(\mathrm{p} / \mathrm{p}) \\
\end{array}$ & $\begin{array}{c}\text { P11- } \\
\text { P12\% } \\
(\mathrm{p} / \mathrm{p}) \\
\end{array}$ & $\begin{array}{c}\text { P13- } \\
\text { P14\% } \\
(\mathrm{p} / \mathrm{p})\end{array}$ & $\begin{array}{c}\text { P15- } \\
\text { P16\% } \\
(\mathrm{p} / \mathrm{p}) \\
\end{array}$ & $\begin{array}{c}\text { P17- } \\
\text { P18\% } \\
(\mathrm{p} / \mathrm{p}) \\
\end{array}$ & $\begin{array}{c}\text { P19- } \\
\text { P20\% } \\
(\mathrm{p} / \mathrm{p})\end{array}$ \\
\hline Caryocar brasiliense fruit oil & 10,00 & 10,00 & 10,00 & 10,00 & 10,00 & 10,00 & 10,00 & 10,00 & 10,00 & 10,00 \\
\hline $\begin{array}{l}\text { Acrylates/C10-30 Alkyl } \\
\text { Acrilate Crosspolymer }\end{array}$ & 0,30 & 0,30 & 0,30 & 0,30 & 0,30 & 0,30 & 0,30 & 0,30 & 0,30 & 0,30 \\
\hline BHT & 0,05 & 0,05 & 0,05 & 0,05 & 0,05 & 0,05 & 0,05 & 0,05 & 0,05 & 0,05 \\
\hline Caprylic/ Capric T & 3,00 & 3,00 & 3,00 & 3,00 & 3,00 & 3,00 & 3,00 & 3,00 & 3,00 & 3,00 \\
\hline Oleyl & 2,00 & 2,00 & 2,00 & 2,00 & 2,00 & 2,00 & 2,00 & 2,00 & 2,00 & 2,00 \\
\hline Dissodium EDTA & 0,10 & 0,10 & 0,10 & 0,10 & 0,10 & 0,10 & 0,10 & 0,10 & 0,10 & 0,10 \\
\hline tolamine (sc & 0,60 & 0,60 & 0,60 & 0,60 & 0,60 & 0,60 & 0,60 & 0,60 & 0,60 & 0,60 \\
\hline $\begin{array}{l}\text { Methyldibromo Glutaronitrile } \\
\text { (and) phenoxyethanol }\end{array}$ & 0,20 & 0,20 & 0,20 & 0,20 & 0,20 & 0,20 & 0,20 & 0,20 & 0,20 & 0,20 \\
\hline Aqua q.s.p. & 100,00 & 100,00 & 100,00 & 100,00 & 100,00 & 100,00 & 100,00 & 100,00 & 100,00 & 100,00 \\
\hline Parfum & 1,00 & 1,00 & 1,00 & 1,00 & 1,00 & 1,00 & 1,00 & 1,00 & 1,00 & 1,00 \\
\hline Carbomer & & 0,20 & - & 0,20 & & 0,20 & & 0,20 & & 0,20 \\
\hline Chloride & & & $\begin{array}{l}\mathrm{X} 1, \mathrm{X} 2 \\
\mathrm{X} 3\end{array}$ & $\begin{array}{l}\mathrm{X} 1 \\
\mathrm{X} 2, \mathrm{X} 3\end{array}$ & 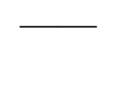 & - & 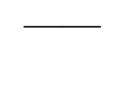 & & 0,10 & 0,10 \\
\hline $\begin{array}{l}\text { Magnesium Sulfate } \\
\text { Sorbitan Oleate }\end{array}$ & $\bar{z}$ & & 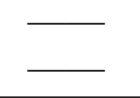 & 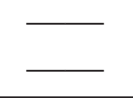 & $\underline{\mathrm{Y} 1-\mathrm{Y} 2}$ & $\underline{Y 1-Y 2}$ & $\overline{\mathrm{Y} 1-\mathrm{Y} 2}$ & $\overline{\mathrm{Y} 1-\mathrm{Y} 2}$ & $\overline{\mathrm{Y} 1-\mathrm{Y} 2}$ & $\overline{\mathrm{Y} 1-\mathrm{Y} 2}$ \\
\hline
\end{tabular}

Onde: $\mathrm{X} 1, \mathrm{X} 2$ e X3 correspondem respectivamente às concentrações de 0,10; 0,15 e 0,20. Y1=0,20 e Y2=0,40. A substituição destes na tabela resulta em diferentes formulações. 
2D) na faixa de temperatura de 40,0 a $80,0^{\circ} \mathrm{C}$. Programouse o aumento da temperatura de $5,0 \pm 1,0$ em $5,0 \pm 1,0{ }^{\circ} \mathrm{C}$, mantendo-se por trinta minutos em cada temperatura. As leituras foram realizadas ao término de $80,0^{\circ} \mathrm{C}$ após o arrefecimento natural das amostras à temperatura ambiente (Braconi et al., 1995).

\section{Ciclo gela-degela}

As amostras foram submetidas à temperatura de $4,0 \pm 2,0{ }^{\circ} \mathrm{C} / 24$ horas (Geladeira Consul, Mod. CFC 28A) e $45,0 \pm 2,0^{\circ} \mathrm{C} / 24$ horas (Estufa Fabbe Primar Ltda, Mod. $171 / 6000 \mathrm{~W})$, completando assim um ciclo. As leituras foram realizadas antes do início do teste e no final do $6^{\circ}$ ciclo (12 dias) (ANVISA, 2004; Ferrari, 1998, 2002).

Determinação do valor do $\mathrm{pH}$ : foi determinado em peagômetro (LOGEN -Mod. LS300-01) inserindo o eletrodo diretamente na diluição aquosa $1: 10(\mathrm{p} / \mathrm{p})$ das amostras (Davis, 1977). Este teste foi realizado em triplicata.

Determinação da condutividade elétrica: Utilizando o condutivímetro (Alpax-ACA-150) aferido com solução padrão, foi avaliada a condutividade elétrica das emulsões à temperatura de $25^{\circ} \mathrm{C}$ inserindo o eletrodo diretamente na amostra (Davis, 1977; Latreille, Paquin, 1990). Este teste foi realizado em triplicata.

\section{Testes de Estabilidade Acelerada}

Sessenta gramas $(60,0 \mathrm{~g})$ das emulsões consideradas estáveis pelos testes preliminares foram submetidos a condições variáveis de temperatura: $4,0 \pm 2,0^{\circ} \mathrm{C}$ (Geladeira Consul Compacto 120 litros), 25, $0 \pm 2,0^{\circ} \mathrm{C}$ (Temperatura Ambiente Controlada), $37,0 \pm 2,0^{\circ} \mathrm{C}$ com Umidade Relativa (UR) de 75,0 $\pm 5,0 \%$ (Câmara Climática Nova Ética 420 - CLD 300) e $45,0 \pm 2,0^{\circ} \mathrm{C}, 75 \pm 5 \%$ UR (Câmara Climática Nova Ética 420 - CLD 300) (ANVISA, 2004; Ferrari, 1998; 2002).

As leituras foram realizadas antes do início do teste (24 h após o preparo das formulações) e no $7^{\circ}, 15^{\circ}, 30^{\circ}$, $60^{\circ}, 90^{\circ}$ dias. Os parâmetros avaliados foram: características organolépticas, valor do $\mathrm{pH}$, determinação da viscosidade e do comportamento reológico.

Determinação da viscosidade e comportamento reológico: realizados em Reômetro (Brookfield-modelo RV-III) tipo cone e placa, acoplado a um Software Rheocalc versão V3.0, utilizando o spindle CP 52 e 0,5 g de amostra a $25,0{ }^{\circ} \mathrm{C}$ (Ferrari, 2002). As medidas foram feitas a velocidades de rotação progressivamente mais altas (1-50 rpm com variação em intervalo de $10 \mathrm{em} 10 \mathrm{rpm}$ ) para obter-se a curva ascendente, e o procedimento foi repetido no sentido inverso com velocidades progressivamente mais baixas ( $50-1 \mathrm{rpm})$ para obter-se a curva descenden- te. Nestas condições, foi atingida uma variação de taxa de deformação de $2-1001 / \mathrm{s}$. As avaliações das amostras foram realizadas no $1^{\circ}, 7^{\circ}, 15^{\circ}, 30^{\circ}, 60^{\circ}$ e $90^{\circ}$ dias.

\section{Análise dos resultados}

Os resultados dos testes de estabilidade acelerada foram submetidos aos testes estatísticos de Tamhane (Sokal, Rohlf, 1995) para amostras heterogêneas e ao teste de Tukey (Sokal, Rohlf, 1995) para as homogêneas. As análises estatísticas foram avaliadas pelo programa SPSS 13.0 for Windows.

\section{RESULTADOS E DISCUSSÃO}

Além de fazer parte da biodiversidade brasileira e estar presente no cerrado mato-grossense, o óleo de pequi apresenta em sua composição química diferentes ácidos graxos, tendo como destaque o oléico $(50,2 \%)$ e o palmítico $(44,3 \%)$, e em menores quantidades os ácidos graxos mirístico, palmitoléico, esteárico, linoléico e linolénico (Croda do Brasil, 2002; Faciole, Gonçalves, 1998). Os ácidos graxos constantes neste óleo são bastante semelhantes aos apresentados na epiderme (Rieger, 1987), o que possibilita seguir mais uma tendência cosmética que é o uso de produtos biomiméticos, favorecendo a compatibilidade formulação-pele.

A determinação da concentração do óleo de pequi (10 $\%-\mathrm{p} / \mathrm{p}$ ) na formulação foi fundamentada nos trabalhos desenvolvidos por Ferrari (2002) e Lima et al. (2006) que obtiveram emulsões estáveis com óleo de andiroba e de babaçu respectivamente nesta concentração. Silva (1994) desenvolveu emulsões do tipo O/A utilizando concentrações de 5,10 e $30 \%$ do óleo de pequi.

Foi proposta uma fórmula base (P1 - Tabela I) e a partir desta, outras diferentes formulações alterando os aditivos e suas respectivas concentrações, caracterizando o desenvolvimento da emulsão.

A fórmula P1 apresentou-se com aspecto de creme, macroscopicamente estável e com cor e odor característicos do óleo de pequi.

Vários fatores podem comprometer a estabilidade físico-química e microbiológica de um sistema emulsionado: escolha de constituintes incompatíveis, tipo e concentração de emulsificantes, velocidade de agitação, tempo de aquecimento e arrefecimento, quantidades das fases, temperatura e ambiente de estocagem e contaminação por microrganismos (Alvarez et al., 2007; Idson, 1993a; Rieger, 1996; Schueller, Romanowski, 2000; Silva, Soares, 1996).

O tensoativo polimérico Acrylates/C10-30 Alkyl Acrilate Crosspolymer, atua pelo mecanismo de estabilização eletroestérica, o que representa uma combinação 
da repulsão elétrica e estabilização estérica (Hemker, 1990; Lochead et al., 1986). Os tensoativos poliméricos apresentam a vantagem de promover estabilidade em baixas concentrações e boa compatibilidade com a pele (Simovic et al., 1999; Tadros et al., 2004).

Assim como Tadros et al. (2004) foram obtidas emulsões estáveis macroscopicamente com baixa concentração de tensoativo $(0,3 \%-\mathrm{p} / \mathrm{p})$ quando comparado aos tensoativos clássicos utilizados como os álcoois graxos etoxilados que requerem uma concentração em torno de $5 \%$ para evitar a coalescência. Os mesmos autores relataram que a estabilidade das emulsões com tensoativos poliméricos está diretamente relacionada com a sua estrutura química e conformação na formação da película interfacial.

Para obter emulsões estáveis com o óleo de pequi, Silva (1994) utilizou diferentes concentrações e misturas de Span $80^{\circledR}(3,5$ e $7 \%)$ e de Tween $80^{\circledR}(5,8,10$ e $15 \%)$, enquanto que no estudo apresentado foram utilizados 0,3 $\%(\mathrm{p} / \mathrm{p})$ de tensoativo, demonstrando que a utilização de tensoativo polimérico pode reduzir consideravelmente a concentração necessária para se obter a estabilidade de um sistema emulsivo.

A dispersão do polímero em estudo pode ser realizada pela adição do mesmo na fase aquosa (método direto) ou na fase oleosa (método indireto) (Pemulen, 1999). Além da facilidade da dispersão, foram obtidas emulsões macroscopicamente mais estáveis quando utilizado o método indireto. Portanto, todas as emulsões estudadas foram manipuladas por este método.

Todas as emulsões preparadas foram do tipo O/A, confirmando a característica hidrofílica do tensoativo (Pemulen, 1999).
Após 24 horas de preparo, foi realizado o teste de centrifugação. Somente as emulsões estáveis neste teste foram submetidas às outras condições de tensão, como estresse térmico e ciclo gela-degela, caracterizando a estabilidade preliminar (Tabela II).

A adição do Carbomer (P2 - Tabela I) manteve a estabilidade da fórmula base (P1) demonstrando compatibilidade deste com o Acrylates/C10-30 Alkyl Acrilate Crosspolymer. Este carbômero atua como estabilizante, aumentando a viscosidade da fase externa evitando a coalescência (Pemulen, 1999).

As formulações P3, P4, P 5, P9, P 10, foram aditivadas com o sodium chloride $(\mathrm{NaCl})$ e magnesium sulfate $\left(\mathrm{MgSO}_{4}\right)$ respectivamente, mas todas apresentaram-se intensamente modificadas com separação de fases após o teste de centrifugação.

Segundo Mishra e Pandit (1989) o $\mathrm{NaCl}$ provoca rearranjo dos tensoativos nas interfaces $\mathrm{O} / \mathrm{Ae} \mathrm{A} / \mathrm{O}$, tornando-as mais rígidas, formando uma barreira mecânica e aumentando a estabilidade. Com o aumento da concentração desse eletrólito as interfaces podem condensar-se e desestabilizar o sistema. Torres et al. (2007) relataram o aumento da estabilidade de emulsões O/A com baixa concentração de $\mathrm{NaCl}$. A adição deste eletrólito também pode interferir nas propriedades reológicas do sistema (Martínez, Riscado, Franco, 2007).

Yang et al. (2006), relataram que o aumento da concentração do $\mathrm{NaCl}$ promove diminuição do potencial zeta $\mathrm{e}$ do tamanho das partículas e conseqüentemente maior estabilidade aos sistemas emulsionados, mas isto não foi observado nas formulações estudadas, pois o aumento da concentração do mesmo levou à instabilidade das formulações.

Assim como Ferrari (2002) foi observada a instabi-

TABELA II - Resultados dos testes preliminares de estabilidade das formulações contendo óleo de pequi.

\begin{tabular}{lccccccc}
\hline Avaliações & $\mathrm{P} 1$ & $\mathrm{P} 2$ & $\mathrm{P} 6$ & $\mathrm{P} 13$ & $\mathrm{P} 14$ & $\mathrm{P} 15$ & $\mathrm{P} 16$ \\
\hline Tempo Inicial $(24 \mathrm{~h})$ & $\mathrm{N}$ & $\mathrm{N}$ & $\mathrm{N}$ & $\mathrm{N}$ & $\mathrm{N}$ & $\mathrm{N}$ & $\mathrm{N}$ \\
Centrifugação & 6,7 & 6,3 & 5,8 & 6,8 & 6,7 & 6,4 & 6,0 \\
Valor do pH & 0,92 & 0,96 & 2,80 & 0,94 & 0,66 & 0,93 & 0,68 \\
Condutividade elétrica $\left(\mathrm{ms} / \mathrm{cm}^{3}\right)$ & & & & & & & \\
Após Estresse Térmico & $\mathrm{N}$ & $\mathrm{N}$ & $\mathrm{N}$ & $\mathrm{N}$ & $\mathrm{N}$ & $\mathrm{N}$ & $\mathrm{N}$ \\
Centrifugação & 6,7 & 6,2 & 5,7 & 6,8 & 6,8 & 6,4 & 6,0 \\
Valor do pH & 0,96 & 1,03 & 3,30 & 1,18 & 1,25 & 1,19 & 1,24 \\
Condutividade elétrica $\left(\mathrm{ms} / \mathrm{cm}^{3}\right)$ & & & & & & & $\mathrm{N}$ \\
Após Ciclo Gela-Degela & $\mathrm{N}$ & $\mathrm{N}$ & $\mathrm{N}$ & $\mathrm{N}$ & $\mathrm{N}$ & $\mathrm{N}$ & $\mathrm{N}$ \\
Centrifugação & 6,7 & 6,2 & 5,7 & 6,8 & 6,8 & 6,4 & 6,0 \\
Valor do pH & 0,94 & 1,00 & 2,90 & 0,94 & 0,98 & 0,93 & 0,94 \\
Condutividade elétrica $\left(\mathrm{ms} / \mathrm{cm}^{3}\right)$ & & & & & & & \\
\hline Onde: $\mathrm{N}=\mathrm{Non}$
\end{tabular}

Onde: $\mathrm{N}=$ Normal. 
lidade do sistema quando adicionado o magnesium sulfate com o objetivo de melhorar a estabilidade da emulsão. A separação de fases segundo este autor pode ter ocorrido devido à incompatibilidade do tensoativo utilizado com o aditivo. Gotchev et al. (2007) obtiveram emulsões estáveis com magnesium sulfate e relataram que a estabilidade foi decorrente da compatibilidade deste com a estrutura química do tensoativo.

Em contrapartida, Tadros et al. (2004) obtiveram emulsões estáveis com altas concentrações de $\mathrm{NaCl}$ e com baixas de $\mathrm{MgSO}_{4}$. Esta estabilidade foi conferida pela presença de polifrutose na estrutura do tensoativo polimérico. Os mesmos relataram que este tipo de estabilidade não é comumente observada em tensoativos que apresentam poli óxido de etileno.

De acordo com a literatura técnica (Noveon, 1997) o carbômero é vulnerável a íons. $\mathrm{O} \mathrm{NaCl}$ modifica o polímero, diminuindo as ligações de hidrogênio e aumenta a fluidez da emulsão podendo provocar a instabilidade. No entanto a adição do Carbomer na formulação P6 (0,1 $\%-\mathrm{p} / \mathrm{p}-\mathrm{NaCl}$ ) promoveu estabilidade do sistema. O mesmo foi observado por Masmoudi et al. (2005).

A utilização do sorbitan oleate é recomendada (Pemulen, 1999) para diminuir o tamanho dos glóbulos de emulsões contendo Acrylates/C10-30 Alkyl Acrilate Crosspolymer, promovendo maior estabilidade do sistema.

As fórmulas P14 e P16 (Tabela II) apesar de não terem apresentado modificações no teste de centrifugação, apresentaram alterações de condutividade elétrica tanto no estresse térmico quanto no ciclo gela e degela, desqualificando-as para os testes de estabilidade acelerada.

As fórmulas $\mathrm{P} 6, \mathrm{P} 13$ e P15, apresentaram resultados considerados estáveis em relação ao primeiro dia tanto no teste de centrifugação quanto no ciclo gela-degela, apresentando alteração na condutividade elétrica apenas no teste de estresse térmico. Masmoudi et al. (2005) relataram que é difícil avaliar a velocidade de estabilização da emulsão somente pela condutividade elétrica, porque não há uma relação linear entre o aumento da condutividade e o fenômeno de instabilidade.

As formulações com o óleo de pequi (P1, P2, P6, P13 e P15 - Tabela II) foram consideradas estáveis nas condições padronizadas dos testes preliminares.

Estatisticamente as formulações P1 e P6 apresentam diferenças significativas $(\mathrm{p}<0,05)$ no valor do $\mathrm{pH}$ durante os 90 dias em tempos e temperaturas diferentes, sendo indicativo de processo de instabilidade. Segundo Masmoudi et al. (2005) a diminuição do $\mathrm{pH}$ pode representar uma oxidação da fase oleosa com formação de hidroperóxidos ou mesmo a hidrólise de triglicerídeos levando à formação de ácidos graxos.
As análises reológicas em função da temperatura são fundamentais para obter informações da estabilidade física e consistência do produto. A reologia da emulsão é uma manifestação direta da interação das forças que ocorrem no sistema. Os diferentes processos que ocorrem na emulsão como a cremeação e sedimentação, floculação e coalescência podem ser investigados através da reologia (Mostefa et al., 2006; Tadros, 1999; 2004).

$\mathrm{O}$ índice de consistência representa a viscosidade do produto e é calculado através de modelos matemáticos adequados ao comportamento reológico (Martin, 1993). Após análises estatísticas as formulações P6, P13 e P15 apresentaram diferença significativa e aumento no índice de consistência em diferentes tempos e temperaturas, sendo então, excluídas do estudo, entendendo que estas alterações representam um processo de instabilidade.

Em todas as formulações citadas acima, a viscosidade aumentou no final dos 90 dias. De acordo com Leonardi et al. (2000) o aumento da viscosidade apresentado pode ser decorrente da evaporação da água das formulações.

Os resultados desta pesquisa também estão de acordo com os obtidos por Guaratini, Gianeti e Maia Campos (2006), cujas formulações estudadas apresentaram aumento no índice de consistência.

Na Figura 1 pode-se observar o perfil do $\mathrm{pH}$ durante os 90 dias de estocagem nas diferentes temperaturas da formulação P2. Apesar da variação e diferença estatística na temperatura de $45,0^{\circ} \mathrm{C}$ no $15^{\circ}$ e $60^{\circ}$ dia, não foi considerada instável pois não apresentou alteração nas caracte-

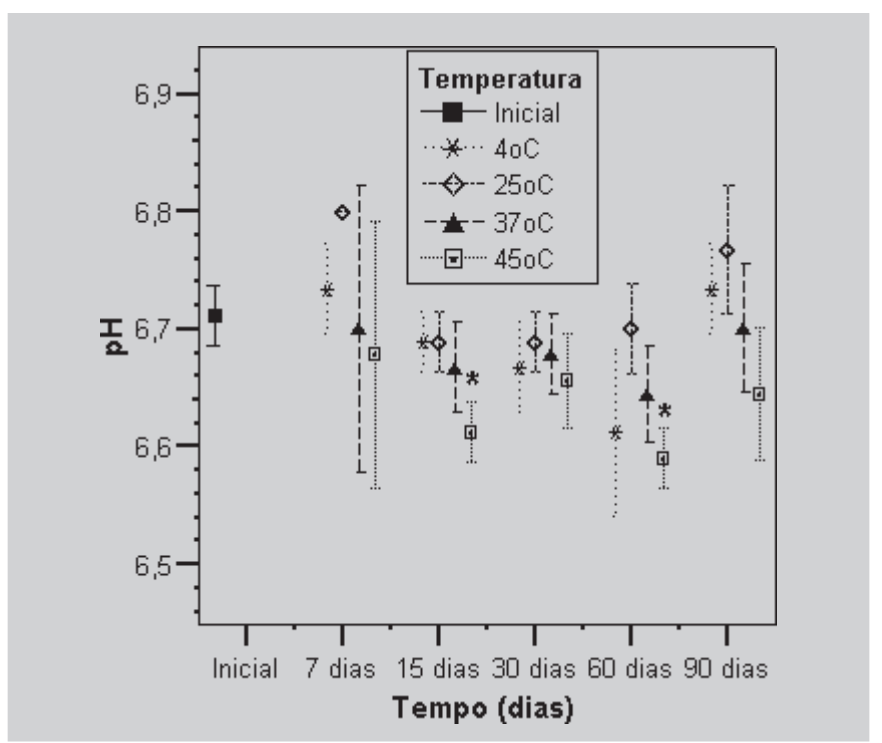

FIGURA 1 - Perfil do $\mathrm{pH}$ durante os testes de estabilidade acelerada da formulação P2. Os resultados foram expressos pela média das leituras $(n=3)$ de cada lote $(n=3)$ e a barra de erro representa a média do intervalo de confiança. * $\mathrm{p}<0,05$. 


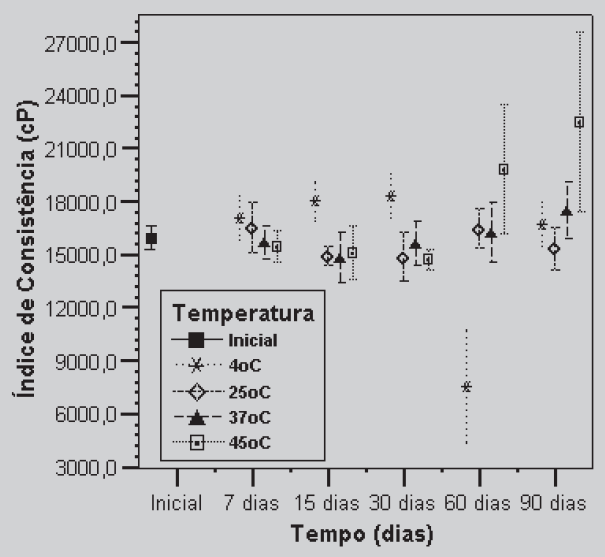

FIGURA 2 - Perfil do índice de consistência durante os testes de estabilidade acelerada da formulação P2. Os resultados foram expressos pela média das leituras $(n=3)$ de cada lote $(n=3)$ e a barra de erro representa a média do intervalo de confiança.

rísticas organolépticas e viscosidade além de manter dentro da faixa do $\mathrm{pH}$ dermatológico (Rieger, 1987).

$\mathrm{O}$ índice de consistência, calculado pelo modelo matemático de Herschel-Bulkley (Figura 2) não apresentou diferença estatística. Estes resultados configuram que não houve alteração da viscosidade do produto durante o tempo de estudo da estabilidade acelerada caracterizando estabilidade da formulação (Ferrari, 2002).

Outros resultados que podem caracterizar a estabilidade da formulação é a viscosidade aparente, que representa a viscosidade do produto a uma dada faixa de taxa de deformação (Martin, 1993). Não houve diferença estatística (Tabela III) quando comparadas às leituras do primeiro e $90^{\circ}$ dia de estocagem nas diferentes condições de temperatura.

Como estatisticamente não apresentou diferença entre as leituras e os lotes, fez se opção por apresentar os reogramas do primeiro e do $90^{\circ}$ dia nas diferentes temperaturas (Figura 3).

Pela análise dos reogramas, pode se verificar que as formulações não obedeceram à lei de Newton sendo então consideradas fluídos não-Newtonianos (Martin, 1993). Observa-se também que a viscosidade é diminuída com o aumento do gradiente de cisalhamento (Figura 3) categorizando um fluído pseudoplástico (Martin, 1993; Tadros, 2004).

Este perfil foi confirmado com o índice de fluxo (Tabela III), também calculado matematicamente, obtido da inclinação da curva de log da tensão de cisalhamento versus o log da velocidade de cisalhamento. Este índice indica o quanto o produto se afasta do perfil Newtoniano. Quando este parâmetro é menor que 1,0 é indicativo de
TABELA III - Valores da viscosidade aparente mínima (cP), índice de fluxo, área de histerese (d/ $\left.\mathrm{cm}^{2} . \mathrm{s}\right)$, da emulsão P2 submetida ao teste de estabilidade acelerada.

\begin{tabular}{lcccc}
\hline $\begin{array}{l}\text { Tempo } \\
(\text { dias })\end{array}$ & $\begin{array}{c}\text { Temperatura } \\
\left({ }^{\circ} \mathrm{C}\right)\end{array}$ & $\begin{array}{c}\text { Viscosidade } \\
\text { aparente } \\
\text { mínima }(\mathrm{cP})\end{array}$ & $\begin{array}{c}\text { Índice } \\
\text { de fluxo }\end{array}$ & $\begin{array}{c}\text { Área de } \\
\text { histerese } \\
\left(\mathrm{d} / \mathrm{cm}^{2} . \mathrm{s}\right)\end{array}$ \\
\hline $1^{\circ}$ & $25^{\circ} \mathrm{C}$ & 1716,51 & 0,44 & 212 \\
& $4^{\circ} \mathrm{C}$ & 1555,77 & 0,45 & $(-) 5776$ \\
$90^{\circ}$ & $25^{\circ} \mathrm{C}$ & 1603,40 & 0,45 & 2130 \\
& $37^{\circ} \mathrm{C}$ & 1520,05 & 0,38 & 6900 \\
& $45^{\circ} \mathrm{C}$ & 1649,04 & 0,35 & 8028 \\
\hline $\mathrm{p}<0,05$. & & & &
\end{tabular}

pseudoplasticidade (Martin, 1993; Ferrari, 2002; Corrêa et al., 2005; Guaratini, Gianeti, Maia Campos, 2006).

O comportamento pseudoplástico é freqüente em formulações que contém gomas naturais ou sintéticas e polímeros (Yasar, Togrul, Arslan, 2007).

A pseudoplasticidade apresentada pela formulação em estudo está coerente com a sua composição e com os resultados de Corrêa et al. (2005) que estudaram o comportamento do Acrylates/C10-30 Alkyl Acrilate Crosspolymer e do Carbomer verificando o comportamento pseudoplástico destes polímeros.

A tixotropia é uma variável da viscosidade dependente do tempo. Este parâmetro fornece informações da capacidade e do tempo necessário para o produto retornar à sua estrutura após a retirada desta tensão. O grau de tixotropia é avaliado pela área resultante entre a curva ascendente e descendente do reograma, denominada histerese (Martin, 1993; Tadros, 2004).

Na dermocosmética esta característica é desejável, pois os produtos tixotrópicos tornam-se mais fluídos, facilitando a espalhabilidade e recuperam a viscosidade original ou parcial, com o término da tensão, evitando assim que o produto flua sobre a pele (Gaspar, Maia Campos, 2003; Leonardi et al., 2000).

A formulação com o óleo de pequi (P2), apresentou características tixotrópicas. Ao verificar o reograma do $1^{\circ}$ dia (Figura 3A- Tabela III), é pouco perceptível a área de histerese, e neste caso não foi considerado tixotropia porque a variação entre a curva ascendente e descendente não ultrapassou $10 \%$ que é um limite de erro do equipamento. Situações diferentes foram apresentadas nos reogramas do $90^{\circ}$ dia (Figura 3B,C,D e E) de estabilidade, pois apresentaram áreas de histerese (Tabela III) consideráveis em relação ao $1^{0}$ dia.

Assim como os resultados apresentados por Ferrari (2002) na temperatura de $4{ }^{\circ} \mathrm{C}$ ocorreu o fenômeno deno- 


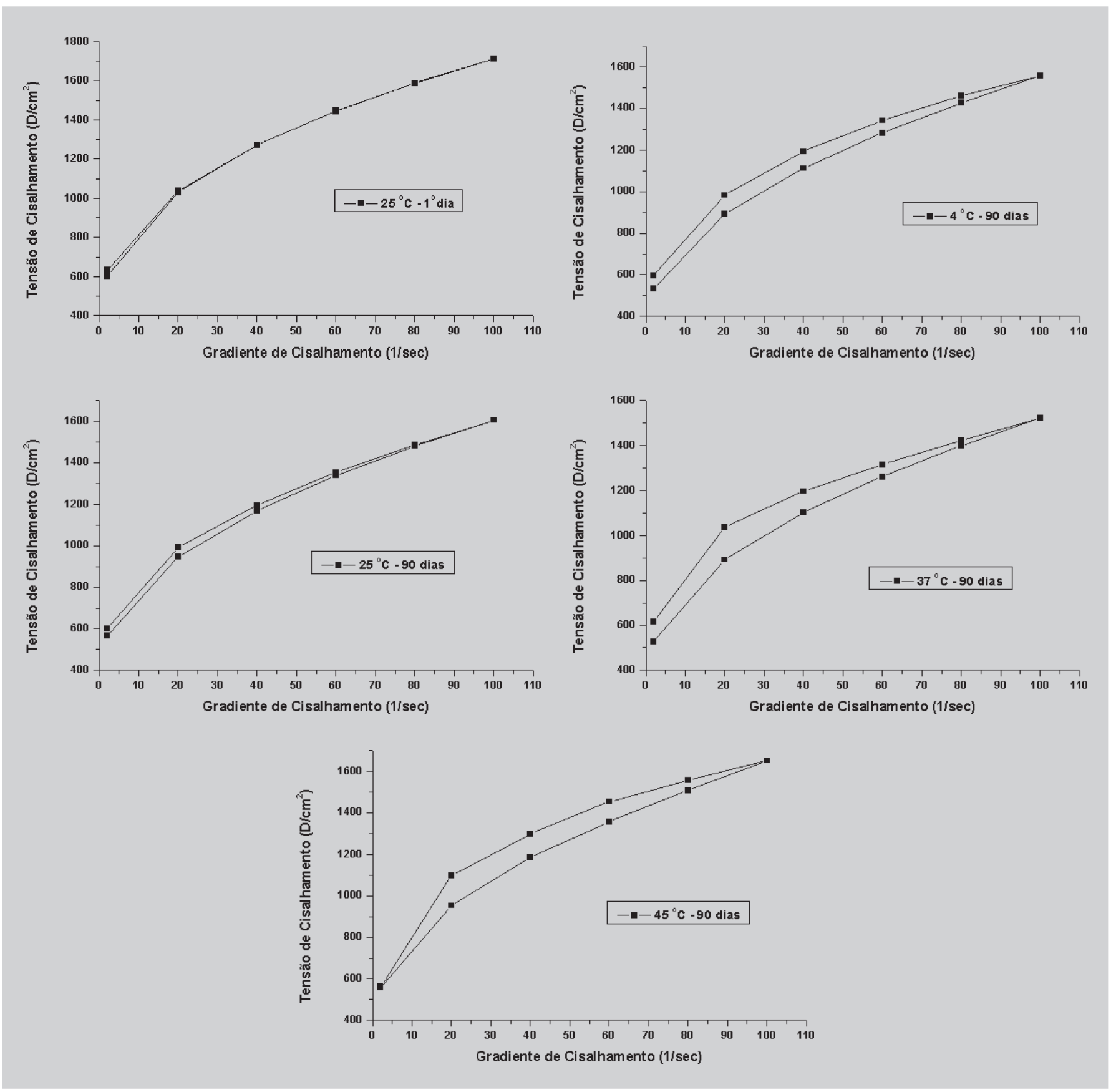

FIGURA 3 - Reogramas das emulsões com óleo de pequi (P2) submetidas aos testes de estabilidade acelerada; 3AReograma após 24 horas $\left(1^{\circ} \mathrm{dia}\right)$ de preparo da emulsão a temperatura de $25^{\circ} \mathrm{C} ; \mathbf{3 B}$ - após 90 dias a temperatura de 4 ${ }^{\circ} \mathrm{C} ; \mathbf{3 C}$ - após 90 dias a temperatura de $25^{\circ} \mathrm{C} ; \mathbf{3 D}$ - após 90 dias a temperatura de $37^{\circ} \mathrm{C}(75 \% \mathrm{UR})$ e $\mathbf{3 E}$ - após 90 dias a temperatura de $45{ }^{\circ} \mathrm{C}(75 \% \mathrm{UR})$.

minado tixotropia negativa ou antitixotropia, ou seja, a viscosidade da curva ascendente foi menor que a da descendente (Tabela III). Esta ocorrência pode ser explicada como resultado do aumento da freqüência de colisão das partículas dispersas ou das moléculas do polímero em suspensão, resultando no aumento da ligação interparticular com o tempo (Martin, 1993). Ainda de acordo com Martin (1993), não deve ser confundido com o fluxo dilatante ou reopexia, pois geralmente estes sistemas apresentam grande quantidade de sólidos dispersos (acima de 50\%).

Uma suposição para esta ocorrência pode ser a própria característica física e composição graxa do óleo de 
pequi, pois abaixo de $25^{\circ} \mathrm{C}$ apresenta cristais e até mesmo consistência semi-sólida. Raphaelides e Georgiadis (2007) relataram que a característica reológica do sistema pode ser alterada na presença de ácidos graxos e seu escoamento afetado pelo tamanho da cadeia graxa e da temperatura.

Sendo assim, pelas análises organolépticas, determinações do valor do $\mathrm{pH}$, da viscosidade e do comportamento reológico durante os testes de estabilidade acelerada, a formulação $\mathrm{P} 2$ pode ser considerada estável.

\section{CONCLUSÕES}

Nas condições estudadas, a emulsão O/A (P2) composta por $10 \%$ de óleo de pequi; $0,3 \%$ de Acrylates/C1030 Alkyl Acrilate Crosspolymer, 0,05\% BHT, 3,0\% de Caprylic/Capric triglyceride; 2,0\% de oleyl alcohol, 0,1 de Disodium EDTA; $0,6 \%$ de solução de Triethanolamine; $0,2 \%$ de Methyldibromo Glutaronitrile (and) phenoxyethanol, 0,2\% de Carbomer; 1,0\% de Parfum e Aqua, foi considerada a que melhor obteve estabilidade.

A partir das avaliações do valor do $\mathrm{pH}$, viscosidade e comportamento reológico durante os testes de estabilidade acelerada pode-se presumir a estabilidade da formulação, apresentando características desejáveis cosmeticamente como a pseudoplasticidade e a tixotropia.

Os resultados também permitem sinalizar mais um óleo do cerrado brasileiro presente na flora mato-grossense com aplicabilidade cosmética para a cadeia produtiva de produtos naturais e desenvolvimento sustentável.

\section{ABSTRACT \\ Use of pequi oil (Caryocar brasiliense) in cosmetics emulsions: development and evaluate of physical stability}

The aims of this study were to development and evaluated the physical stability of $\mathrm{O} / \mathrm{W}$ emulsions containing "Pequi" oil (Caryocar brasiliense). $\mathrm{O} / \mathrm{W}$ emulsions containing $10.0 \%(w / w)$ of Pequi oil were prepared, and to improve the stability, the carbomer, magnesium sulfate, sodium chloride and sorbitan oleate were added and studied. The direction of the emulsions was evaluated by dilution method and by macroscopic analysis, the appearance, homogeneity and organoleptic properties were evaluated. The centrifugation, freeze/defrost cycles and stress thermal were used to investigate the preliminary stability. To evaluate the accelerated stability, the samples were stored at different stress conditions and evaluated the $\mathrm{pH}$ value, macroscopic analysis and rheological behaviour. The $\mathrm{O} / \mathrm{W}$ emulsions prepared with Pequi oil, $0.3 \%$ (w/w) of Acrylates/C10-30 Alkyl Acrilate Crosspolymer and 0.2\% (w/w) of Carbomer have been found to be stables. These presented pseudoplastic flow behaviour and thixotropy. The macroscopic characteristics, the $\mathrm{pH}$ values and the values of the apparent viscosity, both consistency and flow index and hysteresis areas during aging indicated good long-term stability of this formulation.

UNITERMS: Caryocar brasiliense. O/W emulsion. Pequi oil. Rheology. Stability tests.

\section{AGRADECIMENTOS}

Universidade de Cuiabá - UNIC; Fundação de Amparo à Pesquisa do Estado de Mato Grosso - FAPEMAT pelo suporte financeiro; e a Croda do Brasil Ltda pela doação das matérias-primas.

\section{REFERÊNCIAS BIBLIOGRÁFICAS}

ALLEN JUNIOR, L.V. Manipulando emulsões. Int. J. Pharm. Compounding, v.6, n.3, p.168-170,172-174,176, 2004.

ALVAREZ, D.; CASTILLO, M.; PAYNE, F.A.; GARRIDO, M.D.; BANÓN, S.; XIONG, Y.L. Prediction of meta emulsion stability using reflection photometry. J. Food Engineering, v.82, p.310-315, 2007.

ANVISA. Agência Nacional de Vigilância Sanitária. Guia de estabilidade de produtos cosméticos. Brasília: ANVISA, 2004. v.1, 52p.

AZEVEDO-MELEIRO, C.H.; RODRIGUEZ-AMAYA, D.B. Confirmation of the identity of the carotenoids of tropical fruits by HPLC-DAD and HPLC-MS. J. Food Compos. Analysis, v.17, n.3/4, p.385-396, 2004.

BRACONI, F.L.; OLIVEIRA, I.S.; BARONI, M.N.F.; ROCHA FILHO, P.A. Aplicação cosmética do óleo de canola. In: CONGRESSO LATINO AMERICANO E IBÉRICO DE QUÍMICOS COSMÉTICOS, 12. São Paulo, 1995. Anais. São Paulo: Associação Brasileira de Cosmetologia, 1995. p.6-19.

BREUER, M.M. Cosmetics emulsions. In: BECHER, P. (ed.) Encyclopedia of emulsions technology. New York: Marcel Dekker, 1985. v.2, p.385-424. 
COLLEVATI, R.G.; GRATTAPAGLIA, D.; HAY J.D. Evidences for multiple maternal lineages of Caryocar brasiliense populations in the Brazilian Cerrado based on the analysis of chloroplast DNA sequences and microsatellite haplotype variation. Mol. Ecol., v.12, n.1, p.105-115, 2003.

CORRÊA, N.M.; CAMARGO JUNIOR, F.B.; IGNÁCIO, R.F.; LEONARDI, G.R. Avaliação do comportamento reológico de diferentes géis hidrofilicos. Rev. Bras. Cienc. Farm., v.41, n.1, p.73-78, 2005.

CRODA DO BRASIL. Crodamazon Pequi. Campinas: Croda, 2002. 2p. [Catálogo].

DAVIS, H.M. Analysis of creams and lotions. In: SENZEL, A. J. (Ed.). Newburger's manual of cosmetic analysis. Washington: Association of Official Analytical Chemists, 1977. cap.4, p.32.

FACIOLI, N.L.; GONÇALVES, L.A.G. Modificação por via enzimática da composição triglicerídica do óleo de pequi (Caryocar brasiliense Camb). Quím. Nova, v.21, n.1, p.16-19, 1998.

FERRARI, M. Desenvolvimento e avaliação da eficácia fotoprotetora de emulsões múltiplas contendo metoxicinamato de etilexila éleo de andiroba (Carapa guyanensis). Ribeirão Preto, 2002. 142p. [Tese de Doutorado. Faculdade de Ciências Farmacêuticas de Ribeirão Preto. Universidade de São Paulo].

FERRARI, M. Obtenção e aplicação de emulsões múltiplas contendo óleos de andiroba e copaíba. Ribeirão Preto, 1998. 147p. [Dissertação de Mestrado. Faculdade de Ciências Farmacêuticas de Ribeirão Preto. Universidade de São Paulo].

FRANQUILINO, E. Ativos amazônicos. Cosmet. Toiletries, v.18, p.18-24, 26-40, 42-53, 2006.

GASPAR, L.R.; MAIA CAMPOS, P.M.B.G. Rheological behavior and the SPF of sunscreens. Int. J. Pharm., v.250, n.1, p.35-44, 2003.

GOTCHEV, G.; KOLAROV, T.; LEVECKE, B.; TADROS, T.; KHRISTOV, K.; EXEROWA, D. Interaction forces in thin liquid films stabilized by hydrophobically modified inulin polymeric surfactant. 3 . Influence of electrolyte type on emulsion films. J. Surfaces Colloids, v.23, n.11, p.6091-6094, 2007.
GUARATINI, T.; GIANETI, M.D.; MAIA CAMPOS, P.M.B.G. Stability of cosmetic formulations containing esters of Vitamins $\mathrm{E}$ and A: chemical and physical aspects. Int. J. Pharm., v.327, n.1/2, p.12-16, 2006

HEMKER, W.J. Universal oil-in-water polyelectrolyte emulsifiers for advanced cosmetic product formulation. Sofw, v.116, n.14, p.505-508, 1990.

ICID: International Cosmetic Ingredient Dictionary And Handbook. 8.ed. Washington: CTFA, 2000.3v.

IDSON, B. Stability testing of emulsions. Drug Cosmet. Ind., v.103, n.12, p.35-38, 72, 1988.

IDSON, B. Stability testing of emulsions, I. Drug Cosmet. Ind., v.142, n.1, p.27-28, 30, 1993a.

IDSON, B. Stability testing of emulsions, II. Drug Cosmet. Ind., v.142, n.2, p.38, 40, 42-43, 72, 1993 b.

LATREILLE, B.; PAQUIN, P. Evaluation of emulsion stability by centrifugation with conductivity measurements. J. Food Sci., v.55, n.6, p.1666-1668, 1990.

LEONARDI, G.R.; BERALDI, P.; FREITAS, P.C.D.; CAMPOS, P.M.M. Produto de uso tópico com Aloe vera. Cosmet. Toiletries, v.12, n.5, p.44-53, 2000.

LIMA, C.G.; BORSARI, F.O.; CARVALHO, V.F.M.; MOURA, B.A.; PIANOVSKI, A.R.; SILVA, K.K.; SILVA, A.A.S.; VILELA, A.F.G.; FERRARI, M. Utilização do óleo de babaçu em bases dermocosméticas. In: SIMPÓSIO DE PLANTAS MEDICINAIS DO BRASIL, 19, Salvador, 2006. Resumos Anais eletrônicos. Salvador: Dagaz, 2006. 1p.

LOCHEAD, R.Y.; HEMKER, W.J.; CASTANEDA, J.Y.; GARLEN, D. Novel cosmetic emulsions. Cosmet. Toiletries, v.101, n.11, p.125-138, 1986.

MARTIN, A.N. Physical pharmacy. 4.ed. London: Lea \& Febiger, 1993. p.453-473.

MARTIINEZ, I.; RISCARDO, M.A.; FRANCO, J.M. Effect of salt content on the rheological properties of salad dressing-type emulsions stabilized by emulsifier blends. J.Food Engineering, v.80, n.4, p.1272-1281, 2007. 
MASMOUDI, H.; LE DRÉAU, Y.; PICCERELLE, P.; KISTER, J. The evaluation of cosmetic and pharmaceutical emulsions aging process using classical techniques and a new method: FTIR. Int. J. Pharm., v.289, n.1/2, p.117-131, 2005.

MISHRA, B.; PANDIT, J.K. Prolonged release of pentazocine from multiple O/W/O emulsions. Drug Dev. Ind. Pharm., v.15, n.8, p.1217-1230, 1989.

MOSTEFA, N.M.; SADOK, A.H.; SABRI, N.; HADJI, A. Determination of optimal cream formulation from longterm stability investigation using a surface response modeling. Int. J. Cosmet. Sci., v.28, n.3, p.211-218, 2006.

NOVEON. Carbopol ${ }^{\circledR}$ Ultrez Polímero. Informativo técnico. Cleveland: Noveon, 1997. 10p.

PAULA-JUNIOR, W.; ROCHA, F.H.; DONATTI, L.; FADELPICHETH, C.M.T.; WEFFORT-SANTOS, A.M. Leishmanicidal, antibacterial, and antioxidant activities of Caryocar brasiliense Cambess leaves hydroethanolic extract. Rev. Bras. Farmacog., v.16, suppl., p.625-630, 2006.

PEMULEN. Introducing Pemulen ${ }^{\circledR}$ Polymeric Emulsifiers. Tecnical literature. Cleveland: B. F. Goodrich, 1999. 8p.

PINHO, J.J.R.G.; STORPIRTIS S. Formação e estabilidade física das emulsões. Cosmet. Toiletries, v.10, n.6, p.44, 46, 50, 52, 54, 1998.

RAPHAELIDES, S.; GEORGIADIS, N. Effect of fatty acids on the rheological behaviour of pea starch dispersions during heating. Food Hydrocoll., v.21, n.7, p.1188-1200, 2007.

RIBEIRO,H.M. Teoria de estabilidade de emulsões cosméticas. Cosmet. Toiletries, v.14, n.4, p.88-90, 92, 2006.

RIEGER, M.M. Skin lipids and their importance to cosmetic science. Cosmet. Toiletries, v.102, n.7, p.45-49, 1987.

RIEGER, M.M. Teste de estabilidade para macroemulsões. Cosmet. Toiletries, v.8, n.5, p.47-53, 1996.

SCHUELLER, R.; ROMANOWSKI, P. Emulsões. Cosmet. Toiletries, v.12, n.3, p.71-74, 2000.

SHARMA, M.K.; SHAH, D. Introduction to macro - and microemulsions. In: SHAH, D.O. (Ed.). Macro- and microemulsions theory and applications. Washington: American Chemical Society, 1985. cap.1, p. 2.
SILVA, E.C.; SOARES, I.C. Tecnologia de emulsões. Cosmet. Toiletries, v.8, n.5, p.37-46, 1996.

SILVA,E.C. Desenvolvimento de emulsões cosméticas utilizando o óleo de pequi (Caryocar brasiliense Camb). São Paulo, 1994. 112p. [Dissertação de Mestrado. Faculdade de Ciências Farmacêuticas. Universidade de São Paulo].

SIMOVIC, S.; TAMBURIC, S.; MILIC-ASKRABIC, J.; RAJIC, D. An investigation into interactions between polyacrylic polymers and a non-ionic surfactant: an emulsion preformulation study. Int. J. Pharm., v.184, n.2, p.207-217, 1999.

SOKAL, R.R.; ROHLF, F.J. Biometry. 3.ed. New York: W.H. Freeman, 1995. 887p.

TADROS, T.F. Fundamental principles of emulsion rheology and their applications. Colloids and Surfaces A: Physicochem Eng. Aspects, v.91, p.39-55, 1999.

TADROS, T.H.F.; VANDAMME, A.; LEVECKE, B.; BOOTEN, K.; STEVENS, C.V. Stabilization of emulsions using polymeric surfactants based on inulin. Adv. Colloi Interface Sci. v.108/109, p.207-226, 2004.

TADROS, T. Application of rheology for assessment and prediction of the long-tern physical stability of emulsions. Adv. Colloid Interface Sci., v.108/109, p.227-258, 2004.

TORRES, L.G.; ITURBE, R.; SNOWDEN, M.J.; CHOWDHRY, B.Z.; LEHARDE, S.A. Preparation of O/A emulsions stabilized by solid particles and their characterization by oscillatory rheology. Colloids and Surfaces A: Physicochem. Eng. Aspects, v.302, p.439-448, 2007.

USP: United States Pharmacopoeia. 23.ed. Rockville: United States Pharmacopeial Convention, 1990. p.1703-1705.

YANG, J.; WAMER, W.G.; HOWARD, P.C.; BOUDREAU, M.D.; FU, P.P. Levels of retinyl palmitate and retinol in the stratum corneum, epidermis, and dermis of female SKH1 mice topically treated with retinyl palmitate. Toxicol. Ind. Heath, v.22, n.4, p.181-191, 2006.

YASAR, F.; TOGRUL, H.; ARSLAN, N. Flor properties of cellulose and carboxymethyl cellulose from orange peel. J. Food Engineering, v.81, p.187-199, 2007.

Recebido para publicação em 10 de agosto de 2007 Aceito para publicação em 29 de janeiro de 2008 